\title{
AVALIAÇÃO DO PROCESSO DE OBTENÇÃO DE MICRO- ESFERAS DE PARAFINA ATRAVÉS DE METODOLOGIA DE SUPERFÍCIE DE RESPOSTA (MSR)
}

\author{
H. R. TESTAI ${ }^{1}$, K. L. RESENDE ${ }^{1}$, T. J. LOPES $^{2}$, G. A. FERNANDES ${ }^{1}$ \\ ${ }^{1}$ Universidade Federal de Itajubá - UNIFEI, Instituto de Engenharia Mecânica \\ ${ }^{2}$ Universidade Federal do Rio Grande - FURG, Escola de Química e Alimentos \\ E-mail para contato: gersonfernandes@unifei.edu.br
}

\begin{abstract}
RESUMO - Micro-esferas de parafina podem ser usadas para a produção de biomateriais ou arcabouços porosos. Alguns estudos mostram que o tamanho ideal de poros para o crescimento de células, deve ficar entre 50 e $150 \mu \mathrm{m}$. Neste trabalho, micro-esferas de parafina foram produzidas por suspensão, a qual foi mantida em constante agitação (500 e $600 \mathrm{rpm})$. Foram testadas diferentes concentrações de sulfato de sódio (1,5 e 4,5 g/L) e também foram testadas duas concentrações diferentes de PVA, 2 e $6 \mathrm{~g} / \mathrm{L}$, que estabilizam as micro-partículas, evitando sua coalescência. A distribuição de tamanho de partículas obtidas foi determinada por meio de peneiramento. Foram calculados os valores do Diâmetro Linear Médio $\left(\mathrm{d}_{10}\right)$, a Média Superficial dos Diâmetros $\left(\mathrm{d}_{32}\right)$ e a Média Volumétrica dos Diâmetros $\left(\mathrm{d}_{43}\right)$. Os resultados deste trabalho mostraram que o aumento da agitação do sistema diminui o diâmetro médio das partículas, sendo o efeito mais significativo do processo.
\end{abstract}

\section{INTRODUÇÃO}

A parafina é um material que vem sendo utilizado como agente porogênico para produção de biomateriais porosos. Sua importância está relacionada ao fato de ser um material bioinerte, muito acessível, de baixo custo e com baixo ponto de fusão (esta propriedade facilita a formação dos poros, justificando daí o seu uso como material porogênico). O uso de biomateriais porosos está relacionado à substituição ou reconstrução de órgãos ou tecidos danificados por doença ou acidente físico. Para tal finalidade, o agente porogênico deve ser inserido em um suporte, ao qual Ma e Langer (1995) atribuíram o nome de arcabouço ou scaffold. De acordo com Ítala (2001), para uso em tecidos ósseos o agente porogênico deve possuir diâmetro entre 50 e $125 \mu \mathrm{m}$.

São diversos os fatores que interferem no tamanho das microesferas porogênicas, sendo os principais a frequência da agitação, o tipo de reator, formato da hélice, temperatura e a concentração de estabilizantes. A agitação deve ser suficiente para reduzir as partículas em gotículas menores e, simultaneamente, evitar a coalescência das gotas. Maiores velocidades de agitação tendem a produzir partículas com diâmetros menores. Segundo Machado et al. (2007), o estabilizante é responsável por dar a forma esférica às micro-partículas produzidas e por evitar a aglutinação das mesmas. 
O PVA ou poli(álcool vinílico) é um agente estabilizante hidrossolúvel que é adsorvido pela gota de monômero, formando uma fina camada ao seu redor (Schlischting, 2003), como mostra a Figura 1. A parte hidrofóbica do PVA fica voltada para o interior da gota, enquanto a hidrofílica, para o meio aquoso. Logo, quando duas gotas revestidas pelo estabilizante se encontram, as cargas presentes na fina camada, por serem semelhantes, irão se repelir, impedindo a coalescência das gotas (estabilização estérica).

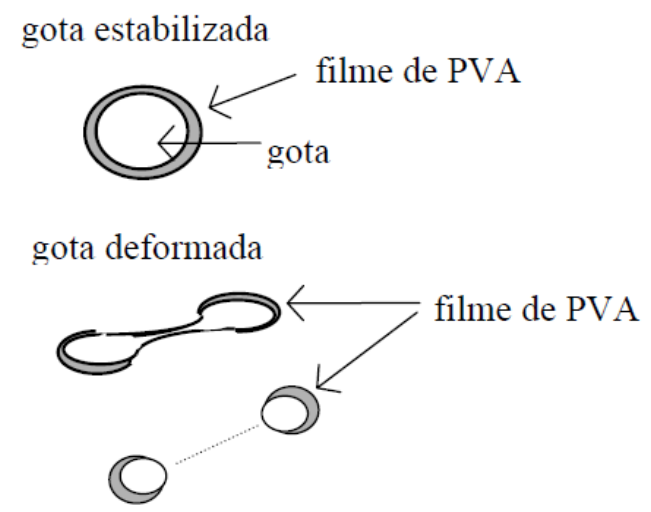

Figura 1- Representação esquemática do estabilizante adsorvido na superfície da gota.

No processo de fabricação das esferas de parafina, o monômero (parafina) é fundido e transformado em pequenas gotas ou pérolas, as quais se encontram na fase líquida. São mantidas sob agitação constante e estabilizadas pelo agente estabilizante e pelo surfactante. A agitação irá contribuir para a diminuição do diâmetro das gotas, pois cria uma zona de cisalhamento próxima à hélice do agitador, quebrando as pérolas em gotículas ainda menores. Depois de formadas as gotas, o sistema é resfriado para que as gotículas se solidifiquem e se tornem micro-partículas de parafina. Esse processo está representado na Figura 2.

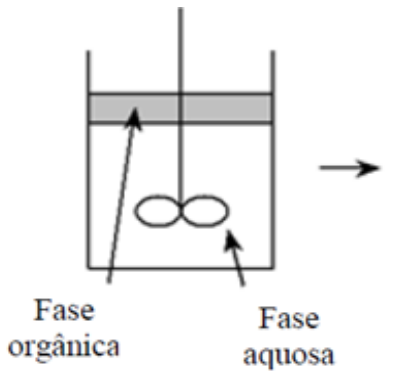

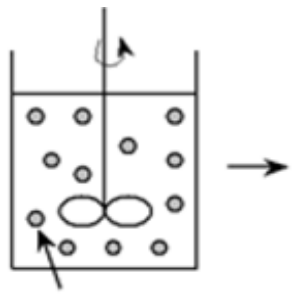

Gotículas de parafina liquida

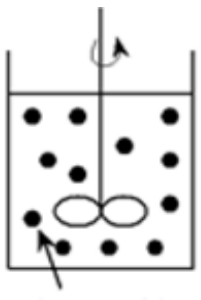

Partículas de parafina

Figura 2 - Etapas da produção das micro-partículas de parafina.

As micro-partículas produzidas passaram por uma análise estatística para determinar seu tamanho e distribuição de diâmetro. Existem vários métodos de análise para determinar o tamanho e a distribuição de tamanho de um sólido particulado. Neste trabalho foram utilizados para a análise de resultados os métodos do Diâmetro Linear Médio $\left(\mathrm{d}_{10}\right)$, Média Superficial dos Diâmetros $\left(\mathrm{d}_{32}\right)$ e da Média Volumétrica dos Diâmetros $\left(\mathrm{d}_{43}\right)$, (Rawle, 2013).

\section{MATERIAL E MÉTODOS}




\subsection{Obtenção das micro-esferas de parafina}

$\mathrm{Na}$ obtenção de parafina na forma de micro-esferas utilizou-se um béquer de vidro $(\mathrm{V}=1 \mathrm{~L})$ contendo $500 \mathrm{~mL}$ de água deionizada, onde foi adicionado diferentes concentrações do estabilizante poli (álcool vinílico) PVA da marca Vetec (4,0 e 12,0 g/L), e adições do coagulante sulfato de sódio (3,0 e 9,0 g/L), marca Vetec. Esta solução foi aquecida a $60^{\circ} \mathrm{C}$ e foi adicionada a parafina comercial (Vetec) e aguardada sua total fusão. Colocou-se o sistema sob agitação mecânica durante 3 min., e então foi realizado um resfriamento rápido. As esferas foram então e lavadas com água destilada e filtradas a vácuo.

Depois de secas a temperatura ambiente as micro-esferas foram separadas por meio de peneiramento em diferentes faixas granulométricas. Foram calculados o diâmetro médio e a dispersão para cada ensaio.

\subsection{Planejamento experimental para o PVA}

O planejamento experimental é uma ferramenta utilizada em muitos processos de análise, formulações de novos sistemas de operações e aprimoramento de sistemas de operações usuais. $\mathrm{Na}$ obtenção da parafina foi avaliada a influência das seguintes variáveis: A) Concentração de Sulfato, B) Concentração de Estabilizante (PVA) e C) Agitação do sistema; com seus respectivos níveis de variação mostrados na Tabela 1 . Foi adotado um planejamento experimental fatorial completo $2^{3}$.

Tabela 1 - Fatores e níveis estudados no planejamento experimental.

\begin{tabular}{|l|c|c|}
\hline \multirow{2}{*}{} & \multicolumn{2}{|c|}{ Níveis codificados } \\
\cline { 2 - 3 } & -1 & +1 \\
\hline Conc. de Sulfato de Sódio (g/L) & 3 & 9 \\
\hline Conc. de Estabilizante (g/L) & 4 & 12 \\
\hline Agitação (rpm) & 400 & 600 \\
\hline
\end{tabular}

Com os resultados obtidos durante a etapa de utilização da matriz experimental $2^{3}$, foi obtido um modelo estatístico empírico, para ser utilizado na previsão do valor da variável resposta no processo estudado $\left(\mathrm{d}_{10}, \mathrm{~d}_{32}, \mathrm{~d}_{43}\right)$, sendo considerada uma variável aleatória y, que se distribui em torno de uma média populacional $\eta\left(x_{1}, x_{2}\right)$, com uma variância populacional $\sigma^{2}\left(x_{1}, x_{2}\right)$ (Equação 1):

$$
y\left(x_{1}, x_{2}\right)=\eta\left(x_{1}, x_{2}\right)+\varepsilon\left(x_{1}, x_{2}\right)
$$

onde $\varepsilon$ é o desvio aleatório com que as observações flutuam em torno da média.

Para este teste estatístico, foi escolhido o modelo com apenas os efeitos principais. Também, foi suposto que os desvios variam segundo uma distribuição normal, sendo que, para o planejamento fatorial completo a média populacional $\eta\left(x_{1}, x_{2}\right)$ pode ser representada por uma combinação linear 
das variáveis x1 e x2 (Equação 2).

$$
\eta\left(x_{1}, x_{2}\right)=\beta_{o}++\beta_{1} x_{1}++\beta_{2} x_{2}
$$

onde $\beta_{0}$ é o valor da média global das respostas e $\beta_{1}$ e $\beta_{2}$ representam os valores populacionais dos efeitos lineares dos efeitos principais e do efeito da interação, por unidade de $\mathrm{x}_{1} \mathrm{e} \mathrm{x}_{2}$.

Os modelos estatísticos testados no ajuste dos valores obtidos experimentalmente serão avaliados segundo análise de variância e coeficiente de determinação. Também, será realizada a localização dos níveis de $\mathrm{x}_{1}, \mathrm{x}_{2}, \ldots, \mathrm{xk}$, que maximizam a resposta estimada (predita). Este ponto, se existir, será um conjunto de $\mathrm{x}_{1}, \mathrm{x}_{2}$, ., $\mathrm{x}_{\mathrm{k}}$ para o qual as derivadas parciais são iguais a zero (Equação 3):

$$
\partial \hat{y} / \partial x_{1}=\partial \hat{y} / \partial x_{2}=\ldots=\partial \hat{y} / \partial x_{k}=0 \text {. }
$$

Após a análise estatística dos coeficientes, a influência dos fatores e suas interações sobre as respostas serão analisadas através a utilização do software Statistica $6.0^{\circledR}$.

\section{RESULTADOS E DISCUSSÃO}

Um planejamento fatorial completo $2^{3}$ foi utilizado para avaliar a influência das variáveis: A) Concentração de Sulfato, B) Concentração de Estabilizante (PVA) e C) Agitação do sistema, com níveis codificados predefinidos, sobre o diâmetro de corte das partículas de parafina. A Tabela 2 mostra a matriz de planejamento completo $2^{3}$ e a as respostas obtidas para cada ensaio.

Tabela 2 - Matriz do planejamento experimental $2^{3}$, com suas respectivas respostas.

\begin{tabular}{|c|c|c|c|c|c|c|}
\hline Ensaio & \multicolumn{3}{|c|}{ Fatores } & \multicolumn{3}{c|}{ Respostas } \\
\cline { 2 - 7 } & $\begin{array}{c}\text { Conc. Sulfato } \\
\text { de Sódio }(\mathrm{g} / \mathrm{L})\end{array}$ & $\begin{array}{c}\text { Conc. de } \\
\text { PVA } \\
(\mathrm{g} / \mathrm{L})\end{array}$ & $\begin{array}{c}\text { Agitação } \\
(\mathrm{rpm})\end{array}$ & $\begin{array}{c}\mathrm{d}_{10} \\
(\mu \mathrm{m})\end{array}$ & $\begin{array}{c}\mathrm{d}_{32} \\
(\mu \mathrm{m})\end{array}$ & $\begin{array}{c}\mathrm{d}_{43} \\
(\mu \mathrm{m})\end{array}$ \\
\hline 1 & $3(-1)$ & $4(-1)$ & $400(-1)$ & 133,471 & 201,437 & 235,174 \\
\hline 2 & $9(+1)$ & $4(-1)$ & $400(-1)$ & 161,713 & 235,210 & 279,454 \\
\hline 3 & $3(-1)$ & $12(+1)$ & $400(-1)$ & 165,494 & 307,260 & 440,700 \\
\hline 4 & $9(+1)$ & $12(+1)$ & $400(-1)$ & 169,775 & 256,362 & 330,040 \\
\hline 5 & $3(-1)$ & $4(-1)$ & $600(+1)$ & 113,197 & 180,573 & 213,195 \\
\hline 6 & $9(+1)$ & $4(-1)$ & $600(+1)$ & 132,279 & 229,360 & 267,519 \\
\hline 7 & $3(-1)$ & $12(+1)$ & $600(+1)$ & 127,751 & 211,624 & 267,141 \\
\hline 8 & $9(+1)$ & $12(+1)$ & $600(+1)$ & 118,980 & 175,586 & 198,231 \\
\hline
\end{tabular}

*(-1) e (+1) são os Níveis de variação dos fatores.

As Figuras 3 e 4 mostram a distribuição de tamanho das micro-esferas com o aumento da 
concentração de sulfato de sódio, utilizando diferentes concentrações de PVA e agitação de 600 rpm.

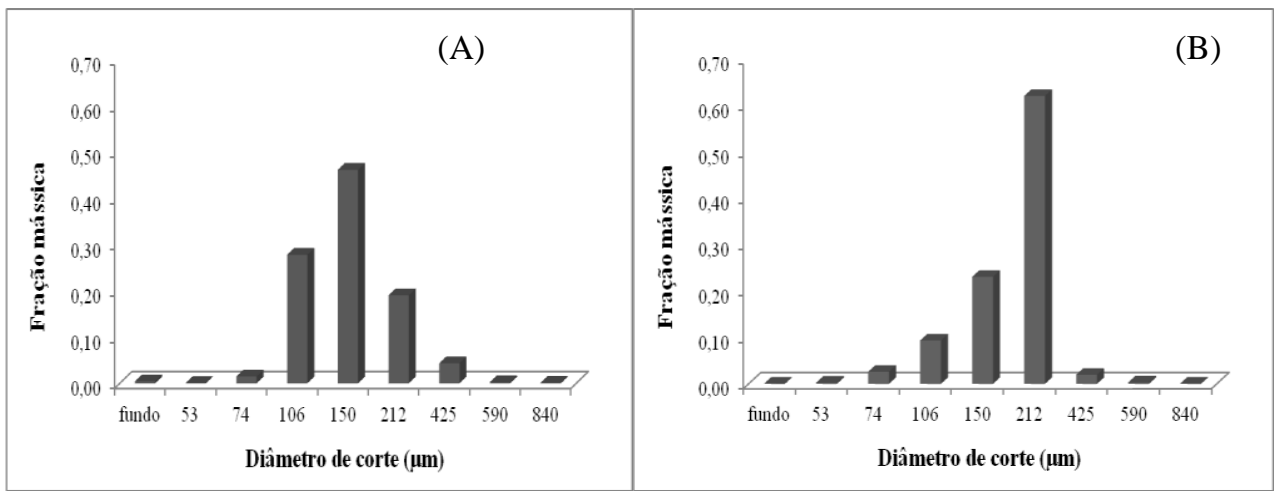

Figura 3: Distribuição de tamanho das partículas para: (A) 3,0 e (B) 9,0 g/L de $\mathrm{Na}_{2} \mathrm{SO}_{4}$; em 4,0 g/L de PVA

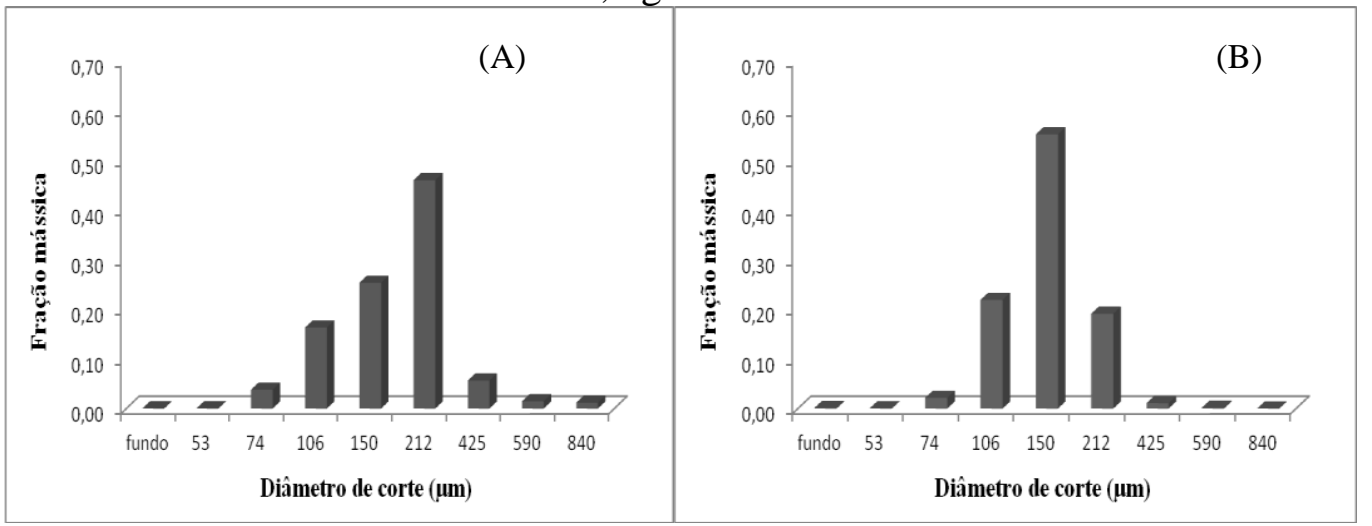

Figura 4: Distribuição de tamanho das partículas para: (A) 3,0 e (B) 9,0 g/L de $\mathrm{Na}_{2} \mathrm{SO}_{4}$; em 12,0 g/L de PVA

As Figuras 5 e 6 mostram a distribuição de tamanho das micro-esferas com o aumento da concentração de sulfato de sódio, utilizando diferentes concentrações de PVA e agitação de 400 rpm.
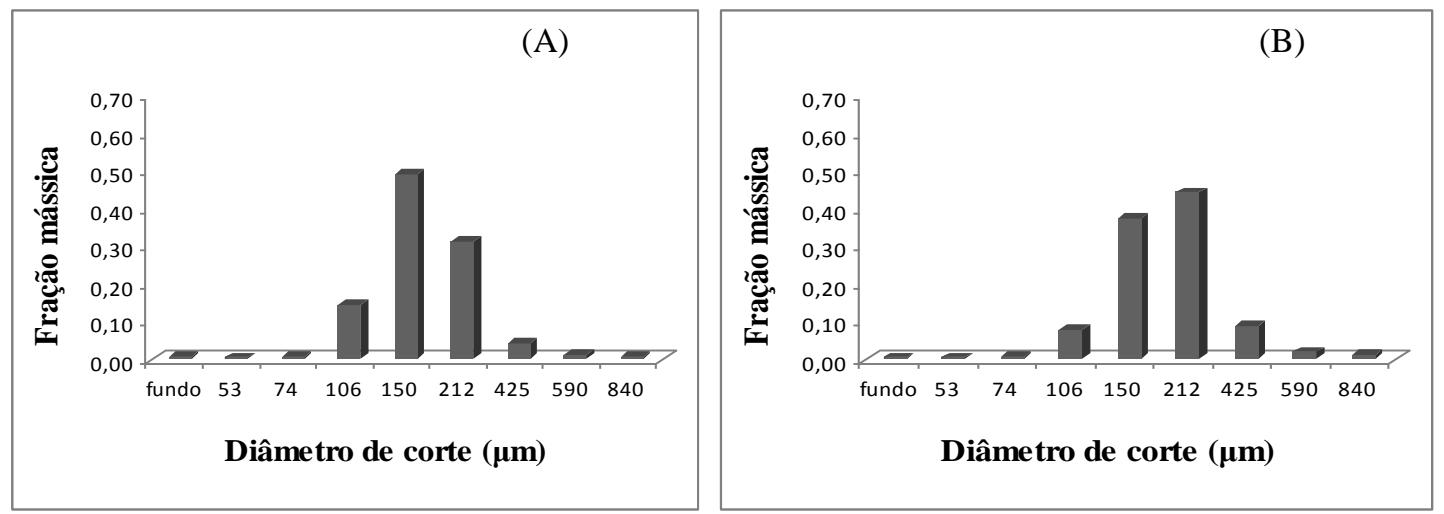

Figura 5: Distribuição de tamanho das partículas para: (A) 3,0 e (B) 9,0 g/L de $\mathrm{Na}_{2} \mathrm{SO}_{4}$; em $4,0 \mathrm{~g} / \mathrm{L}$ de PVA 

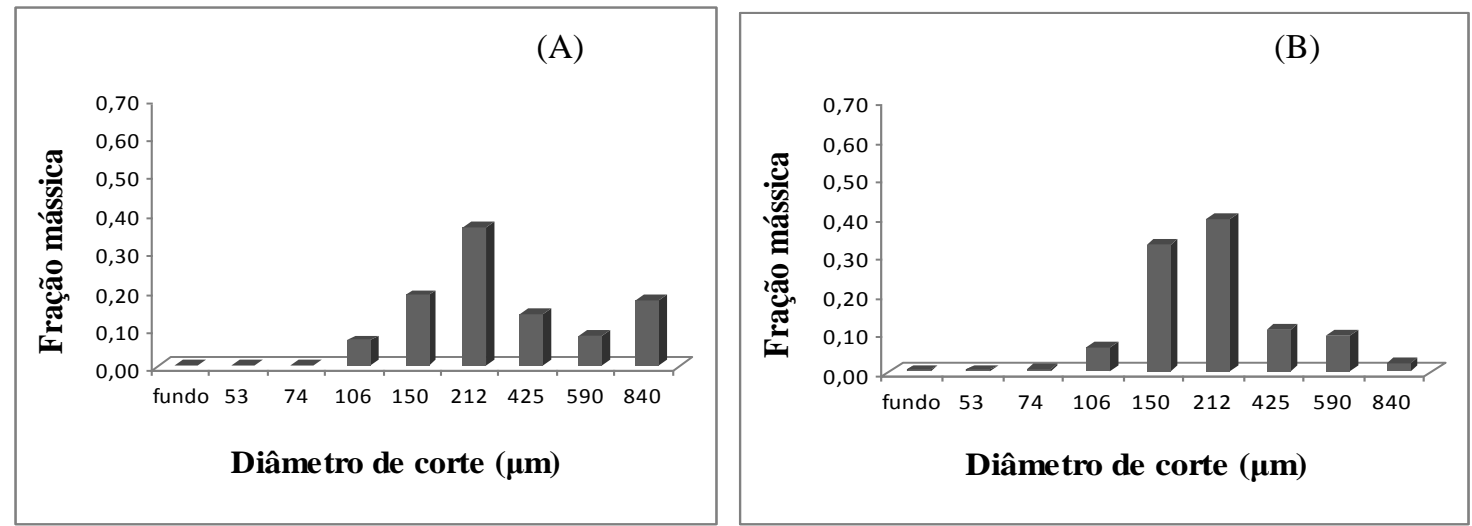

Figura 6: Distribuição de tamanho das partículas para: (A) 3,0 e (B) 9,0 g/L de $\mathrm{Na}_{2} \mathrm{SO}_{4}$; em 12,0 g/L de PVA

Pelos resultados das Figuras 4 a 7, é possível ver que a maioria das micro-esferas ficaram entre 106 e $425 \mu \mathrm{m}$. A distribuição do tamanho das partículas (DTP) tende a ser unimodal.

\subsection{Análise das curvas de nível para $d_{10}, d_{32}$ e $d_{43}$.}

Através da Figura 8,9 e 10 pode-se observar as curvas de nível correspondentes à superfície de resposta gerada pelo modelo empírico para os diâmetros $\mathrm{d}_{10}, \mathrm{~d}_{32} \mathrm{e} \mathrm{d}_{43}$.

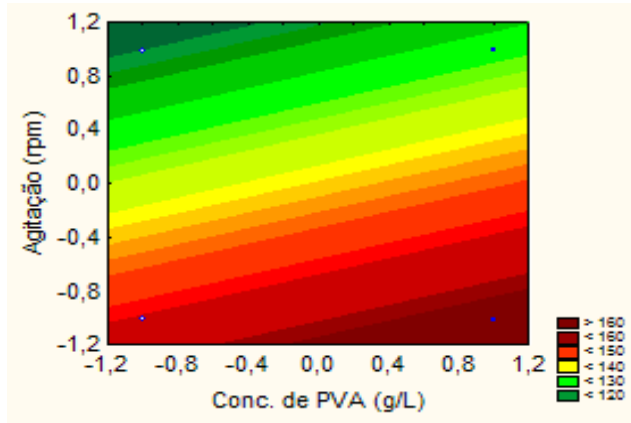

\begin{tabular}{|l|r|r|r|r|}
\hline & Efeito & $\begin{array}{l}\text { Desvio } \\
\text { Padrão }\end{array}$ & $\begin{array}{c}-95 \% \\
\text { Limite de } \\
\text { Confiança }\end{array}$ & $\begin{array}{c}+95 \% \\
\text { Limite de } \\
\text { Confiança }\end{array}$ \\
\hline Média/Interações & 140,33 & 4,29 & 128,43 & 152,23 \\
\hline $\begin{array}{l}\text { (A) Conc. de Sulfato } \\
\text { (g/L) }\end{array}$ & 10,71 & 8,57 & $-13,09$ & 34,50 \\
\hline (B) Conc. de PVA (g/L) & 10,34 & 8,57 & $-13,46$ & 34,13 \\
\hline (C) Agitação (rpm) & $-34,58$ & 8,57 & $-58,36$ & $-10,77$ \\
\hline
\end{tabular}

Figura 8: Curvas de nível para os fatores agitação e Concentração de PVA para o diâmetro $\mathrm{d}_{10}$

(Concentração de Sulfato nível $0=6 \mathrm{~g} / \mathrm{L}$ ).

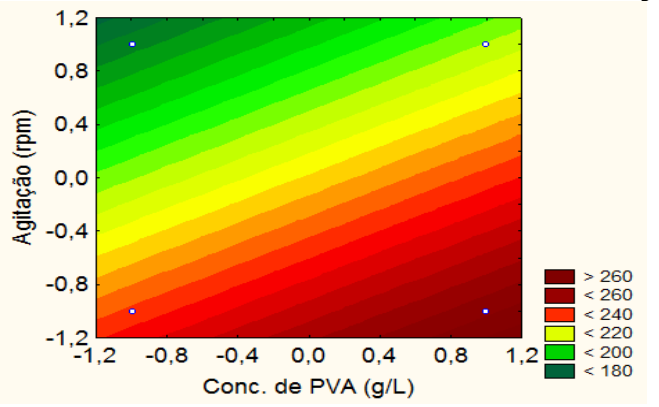

\begin{tabular}{|l|c|c|c|c|}
\hline & Efeito & $\begin{array}{c}\text { Desvio } \\
\text { Padrão }\end{array}$ & $\begin{array}{c}-95 \% \\
\text { Limite de } \\
\text { Confiança }\end{array}$ & $\begin{array}{c}+95 \% \\
\text { Limite de } \\
\text { Confiança }\end{array}$ \\
\hline Média/Interações & 224,68 & 14,26 & 185,09 & 264,26 \\
\hline (A) Conc. de Sulfato (g/L) & $-1,09$ & 28,51 & $-80,26$ & 78,07 \\
\hline (B) Conc. de PVA(g/L) & 26,06 & 28,51 & $-53,10$ & 105,23 \\
\hline (C) Agitação (rpm) & $-50,78$ & 28,51 & $-129,95$ & 28,38 \\
\hline
\end{tabular}

Figura 9: Curvas de nível para os fatores agitação e Concentração de PVA para o diâmetro $\mathrm{d}_{32}$ (Concentração de Sulfato nível $0=6 \mathrm{~g} / \mathrm{L}$ ). 


\section{SCOBRe}

19 a 22 de outubro de 2014

Florianópolis/SC

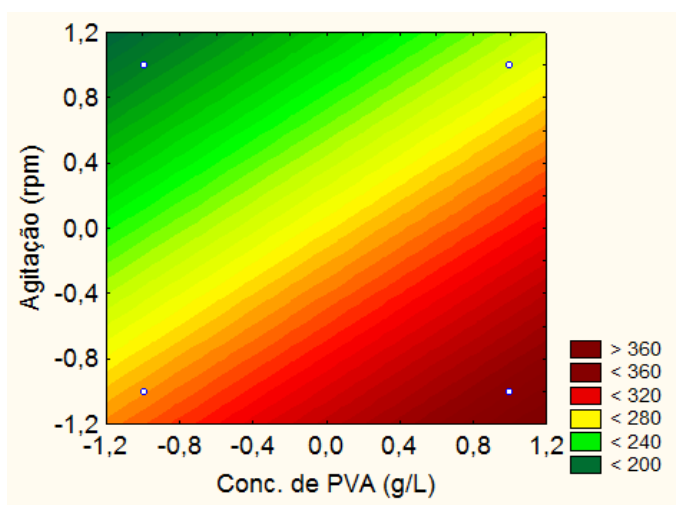

\begin{tabular}{|l|c|c|c|c|}
\hline & Efeito & $\begin{array}{c}\text { Desvio } \\
\text { Padrão }\end{array}$ & $\begin{array}{c}-95 \% \\
\text { Limite de } \\
\text { Confiança }\end{array}$ & $\begin{array}{c}+95 \% \\
\text { Limite de } \\
\text { Confiança }\end{array}$ \\
\hline Média/Interações & 278,93 & 24,59 & 210,67 & 347,20 \\
\hline (A) Conc. de Sulfato (g/L) & $-20,24$ & 49,17 & $-156,77$ & 116,29 \\
\hline (B) Conc. de PVA ' $\mathrm{g} / \mathrm{L}$ ) & 60,19 & 49,17 & $-76,34$ & 196,72 \\
\hline (C) Agitação (rpm) & $-84,82$ & 49,17 & $-221,35$ & 51,71 \\
\hline
\end{tabular}

Figura 10: Curvas de nível para os fatores agitação e Concentração de PVA para o diâmetro $\mathrm{d}_{43}$ (Concentração de Sulfato nível $0=6 \mathrm{~g} / \mathrm{L}$ ).

Nas Figuras 8 a 10, observa-se que o efeito agitação (C) é estatisticamente significativo para o $\mathrm{d}_{10}$, no nível de confiança adotado de $95 \%$, pois o fato do intervalo de confiança não conter o número zero indica que o fator em análise não apresenta efeito nulo, ou seja, ser considerado significativo no nível de confiança testado. Também, foi obtido um valor para o coeficiente de determinação $\left(\mathrm{R}^{2}\right)$ de 0,8281 .

Em relação à análise do sinal algébrico dos efeitos encontrados, estes estão de acordo com o conhecimento que se tem dos fenômenos envolvidos. No que diz respeito à agitação(C) um aumento desta contribui para um menor diâmetro linear médio das partículas presentes no final do processo de obtenção das micro-esferas de parafina. Também é possível verifica, principalmente, que com o aumento da agitação do sistema, o diâmetro das partículas de parafina, após o processo para os 3 diâmetros $\mathrm{d}_{10}, \mathrm{~d}_{32} \mathrm{e} \mathrm{d}_{43}$, encontra-se num nível mínimo na região ótima de processo em agitação 540 a 640 rpm (níveis 0,4 a 1,2). Sendo que para obtenção de partículas maiores, o fator concentração de PVA, se mostra importante juntamente com o fator agitação. $\mathrm{O}$ modelo para o processo de obtenção da parafina para $\mathrm{d}_{10}, \mathrm{~d}_{32}$ e $\mathrm{d}_{43}$ é representado pelas Equações 4, 5 e 6, respectivamente.

$$
\begin{aligned}
& d_{10}=140,33+5,35 A+5,16 B-17,28 C \\
& d_{32}=224,67-0,54 A+13,03 B-25,39 C \\
& d_{43}=278,93-10,12 A+30,096 B-42,41 C
\end{aligned}
$$

onde: "A" é a concentração de sulfato de sódio (g/L); B é a concentração de PVA (g/L) e C é a agitação (rpm).

\section{CONCLUSÕES}

Os ensaios mostraram que o sulfato de sódio não interfere de forma significativamente no filme formado de PVA ao redor das partículas. O aumento do parâmetro de agitação tende a diminuir o diâmetro médio das partículas e o aumento da concentração do sulfato de sódio e de PVA pouco 
afetou o valor.

Os melhores resultados obtidos para o diâmetro das partículas de parafina, para as 3 variáveis dependentes $\mathrm{d}_{10}, \mathrm{~d}_{32}$ e $\mathrm{d}_{43}$, foram obtidos com agitação de 540 a $640 \mathrm{rpm}$ (níveis 0,4 a 1,2). Os valores obtidos para os diâmetros médios lineares $\left(\mathrm{d}_{10}\right)$ e os diâmetros médio de Sauter $\left(\mathrm{d}_{32}\right)$ mostraram a maior quantidade de partículas ideais para serem usadas na fabricação de arcabouços (50 a $125 \mu \mathrm{m})$ (Ítala et al.,2001). Portanto, o processo estudado evidencia e credencia, a possível utilização da parafina obtida via suspensão estabilizada com PVA na produção de arcabouços.

\section{REFERÊNCIAS}

ITALA, A. I.; YLANEN H. O.; EKHOLM C.; KARLSSON K. H.; ARO H. T.. Pore diameter of more than 100 micron is not requisite for bone ingrowth in rabbits. J. Biomed. Mater. Res., 58, p. 679-683, 2001.

MA, P. X.; LANGER, R. Degradation, structure and properties of fibrous nonwoven poly(glycolic acid) scaffolds for tissue engineering. In: Polymers in medicine and pharmacy. p 99-104. R. Mater. Res. Soc., PA, EUA, 1995.

MACHADO, F.; LIMA, E. L.; PINTO, J. C. Uma revisão sobre os processos de polimerização em suspensão. Polímeros, v. 17, n. 2, p. 166-179, 2007.

RAWLE, A. Basic principles of particle size analysis. Disponível em: <http://www.rci.rutgers.edu/ moghe/PSD\%20Basics.pd>. Acesso em: 02 set. 2013.

SCHLISCHTING, Rodrigo. Influência da adição de poliestireno expandido no processo de polimerização do estireno em suspensão. 2003. 105 f. Dissertação (Mestrado em Engenharia Química), UFSC, Florianópolis-SC, 2003. 